\title{
Evaluation of the Efficacy and Safety of 1\% Sodium Chloride (LiceFreee Spray) against 1\% Permethrin Crème Rinse on Head Lice Infested Individuals
}

\author{
Lidia Serrano $^{1}$, Lorraine S. Decesar ${ }^{1}$, Loan Pham $^{2 *}$ \\ ${ }^{1}$ South Florida Family Health and Research Centers, Plantation, USA; ${ }^{2}$ Carmago Pharmaceutical Services, Cincinnati, USA. \\ Email: *LPham@camargopharma.com
}

Received January $21^{\text {st }}, 2013$; revised March $3^{\text {rd }}, 2013$; accepted April $16^{\text {th }}, 2013$

Copyright (c) 2013 Lidia Serrano et al. This is an open access article distributed under the Creative Commons Attribution License, which permits unrestricted use, distribution, and reproduction in any medium, provided the original work is properly cited.

\begin{abstract}
Head lice are a public health issue, and resistance to available over-the-counter pediculicides is a concern. The objective of this randomized study was to evaluate the pediculicidal activity and safety of $1 \%$ Sodium Chloride spray (0.1709 M), (LiceFreee Spray ${ }^{\circledR}$ ) compared to the current recommended treatment for head lice with 1\% Permethrin Crème Rinse. Forty-two subjects were randomized equally into Sodium Chloride or Permethrin group. Products were applied to hair according to the label instructions. After application of the products at Day 1 and Day 8, pediculicidal efficacy and safety were assessed at Day 1, Day 8 and Day 15. Second treatment was only applied on Day 8 to individuals with observed live lice using the same products and protocols as Day 1. Proportion of lice free subjects per group, the reduction in number of live lice per head and adverse effects were recorded after each visit. The results showed significantly higher pediculicidal activity for Sodium Chloride spray (85\%) as compared to Permethrin (45\%) at Day 15 (p < 0.05). Similar numbers of lice per head (21.76 range 10 to 68 versus 21.29 range 10 to 60 for Sodium Chloride and Permethrin groups, respectively) were observed for individuals at Day 1. At Day 15, lice per head infested reduced to $0.55 \pm 1.50$ in Sodium Chloride spray group compared to $5.45 \pm 7.91$ in the Permethrin group $(\mathrm{p}<0.01)$. No serious adverse effects were observed in both groups. Sodium Chloride spray had superior efficacy to $1 \%$ Permethrin Crème Rinse in treating head lice and is a safe and excellent alternative to the current recommended treatment.
\end{abstract}

Keywords: Head Lice; Sodium Chloride; LiceFreee Spray ${ }^{\circledR}$; Nix ${ }^{\circledR}$; Permethrin

\section{Introduction}

Pediculosis capitis caused by Pediculus humanus capitis has been recognized as an issue from the antiquity, and currently remains a common health concern. The traditional perception of head lice as a parasitosis exclusively associated with schoolchildren of low socioeconomic status is challenged by the facts that pediculosis capitis is widespread throughout the world and does not discriminate on socioeconomic status grounds [1,2]. It primarily occurs in pre-school or elementary school age children and those living in the same house, although it can be found in refugees, urban slums, jail inmates, orphanages, and fishing communities [1]. The prevalence worldwide varies from $0.7 \%$ to $59 \%$ and is higher in girls and women. Having a head lice infestation is annoying, can lead to pruritus, sleeplessness, and in extreme cases, ane-

${ }^{*}$ Corresponding author. mia. Secondary bacterial infections can complicate the course of the infestation with Staphylococcus aureus the most commonly implicated pathogen in this setting and can lead to impetigo, cellulitis, pyoderma and abscess formation [3-5]. The consequences of a head lice infestation can also result in psychological frustration for both parents and children. In the USA alone, pediculosis capitis is the most prevalent parasitic infestation of children with 6 - 12 million children infested and needing treatment for head lice each year. Head lice infestation also accounts for 12 - 24 million lost school days and $\$ 4$ - \$8 billion in economic loss due to missed workdays by parents staying home with their children [6].

Parents or non-health care professionals diagnose a head lice infestation by observing a louse crawling on the scalp or more frequently, observation of the nits on the hairs at the nape of the neck or behind the ears [7]. Transmission of head lice is mainly by direct head-to-head 
contact with infested persons, particularly with children in the same school class, team or sharing playground. Fomite routes are less frequent and involve combs, hats, towels, fabrics and warm air movements [8,9].

Removal of the head lice includes traditional physical methods (plucking off head lice etc.), the use of coating gels, oral medication and the application of chemicals to the hair. Using pediculicides is the most frequent method. Pediculicides have shown effectiveness in reducing head lice infestation in populations globally [5]. Normally, pediculicidal treatment has to be applied on two occasions with 7 - 10 days in between, as the first dose of pediculicides primarily kill nymphs and adult lice, while their ovicidal activity is generally poor. The time gap between treatments allows surviving eggs to hatch and the newly hatched nymphs subsequently be killed by the second application [5]. Until recently, topical agents such as permethrin, allethrin, lindane or benzyl benzoate were still among the favorites. However, resistance to pediculicides has been reported in many areas of the United States as well as all around the world [10-12]. For example, reported efficacy of permethrin to head lice was reduced from as high as $97 \%$ - 99\% before 1999 to as low as $10 \%-72 \%$ after 1999 [5]. Importantly, several recent studies have revealed an increasing trend of simultaneous resistance against multiple agents including lindane, phenothrin and permethrin suggesting over-the-counter commercially available insecticidal products may become ineffective $[5,8,10]$. Prescription-only products containing synthetic compounds, such as malathion and carbaryl, have not been established well by controlled trials in term of safety and effectiveness for children less than 6 years old (malathion) or raised the concerns of possible carcinogenic effects (carbaryl) $[10,13]$. However, it has been suggested that resistance is starting to develop to this group also [5,14]. Therefore, alternative drugs for treatment of head lice are needed having the requirements of safety, high effectiveness, available OTC and easy to use.

The primary objective was to compare the efficacy and safety of a one percent solution of Sodium Chloride $(0.1709 \mathrm{M})$, against $1 \%$ Permethrin Crème Rinse, the treatment of choice by the National Guideline Clearinghouse [15], on subjects with head lice immediately following the initial application at 7 (Day 8), and 14 (Day 15) days following the initial application. The study was to also examine the efficacy and safety of the two treatments following one or two applications without the use of the nit comb.

\section{Methods}

\subsection{Study Population}

The study was approved by The Investigational Review
Board Inc. (IRB \# Lf 001-0011) and was conducted in accordance with Good Clinical Practices and with the principles outlined in the Declaration of Helsinki. The study was registered with ClinicalTrials.gov Protocol Registration System ID number NCT01514513 and all study treatments with follow up assessments were performed by the authors listed residing at South Florida Family Health Research Center, 6971 West Sunrise Blvd., Suite 102, Plantation, FL 33,313.

Individuals, aged 4 years or older, having a single place of residence, diagnosed with an active head lice infestation of at least 10 live lice at the Screening visit and a presence of nits were eligible for inclusion in this study. The subjects agreed to not use a lice comb, or any other pediculicides or medicated hair grooming products for the duration of the study (through Day 15 visit). Furthermore, parents and other family members of a child were also screened for head lice. If other household members were found to have head lice and were eligible, they were either enrolled in the study or were treated with the same product and in the same manner as study participants. All the participants of the study and where appropriate their legal guardian were explained the study's procedures and signed informed consents.

Subjects using any form of head lice treatment for at least four weeks or any topical medication for a period of 48 hours prior to the Screening visit (Day 1) were not eligible for inclusion in the study. Individuals were also not eligible for participation if taking systemic or topical medications (including antibiotics), or suffering from visible skin/scalp condition at the treatment sites, of which in the opinion of the investigative personnel would interfere with the evaluation of the test products. Anyone who was allergic or sensitive to ragweed or any ingredient in either test product, pregnant or nursing, who did not understand the subject requirements for study participation and/or exhibited poor compliance with the required visits were also excluded from the study.

Subjects could withdraw from study treatment at any time at their own request, or at the discretion of the investigators for safety, behavioral or administrative reasons.

\subsection{Study Design}

Subjects were randomly assigned by sealed-envelop randomization to receive Sodium Chloride or Permethrin during the treatment period. An equal number of subjects were allocated into each arm of the study. This was an open-label study; as such, the investigators, site personnel and the subjects were aware of the product being used.

Test product was 1\% Sodium Chloride (0.1709 M) 
spray (LiceFreee Spray ${ }^{\circledR}$, in 6 ounce bottles; Lot number J1821 by Tec Laboratories, Inc. Albany, Oregon), and reference product was $1 \%$ Permethrin Crème Rinse $\left(\mathrm{Nix}^{\circledR}\right.$, Insight Pharmaceuticals, LangHorne, PA, in 2 fluid ounce bottles, $280 \mathrm{mg}$ /fluid ounce; Lot number NX0908-Expiration 3/2012). The doses used for test and reference products were strictly followed as outlined on product label directions.

Subjects had the products applied on Day 1 by investigative site personnel after determination of meeting study inclusion criteria. The time of application, the weight of the bottle before and after application, the time elapsed until the hair was considered dry (for Sodium Chloride spray), the start and end time of rinsing (for Permethrin) were recorded.

In the Sodium Chloride spray arm, the product was sprayed on the hair until the hair was completely saturated. The hair was then allowed to dry naturally. Efficacy evaluation was performed 1 hour after the application of Sodium Chloride or earlier if the hair was completely dry using a metal comb. The participants were asked to wait 24 hours before returning to their normal hair hygiene. For those in the Permethrin Crème Rinse treatment group, the hair was washed with baby shampoo (Johnson and Johnson, New Brunswick, NJ) without conditioner and then rinsed with water. The hair was towel dried. Permethrin Crème Rinse was then applied to saturate the hair, scalp, behind the ears and to the nape of the neck. After 10 minutes of treatment with Permethrin Crème Rinse, the hair was rinsed with warm water and towel dried and efficacy of the treatment was assessed using a metal comb. Subjects received treatment at the time they were in the clinic which varied based on appointment time. No nit combs were used during the study. If live lice were observed at the second visit (Day 8), the subjects received a second treatment with the assigned test products following the same procedures mentioned above.

\subsection{Assessment of Efficacy and Safety}

After the initial application of the products on Day 1, evaluation and follow-up visits were performed at day 7 and day 14, respectively, with a \pm 1 day window (Day 8 and Day 15 visit). Demographic parameters such as date of birth, gender, race, ethnicity, size of household, and hair characteristics (length, texture, and curliness) were recorded together with medical history and current medication usage.

During examination, hair and scalp were assessed for the number of live lice at pre-treatment, immediately after the treatment at Day 1 (and if applicable, on Day 8), at Days 8 and 15. The detection of any live lice on a subject was considered a treatment failure with either product used. Numbers of live lice and nits observed in the left, middle, and right side of the head were recorded for all time points but the number of nits was estimated only prior to the course of treatment.

Assessment of other symptoms such as pruritus, erythema, presence of secondary infection, and excoriation was recorded at each visit using a 4-point scale (none 0 , mild 1 , moderate 2 , or severe 3 ). Vital sign measurements such as systolic, diastolic blood pressure and pulse rate, examined after the subject rested in a seated position for at least 5 minutes, and evaluation of existence of red eyes were performed at each visit. Urine pregnancy test was performed at the Screening Visit (Day 1) only to ensure all females of child-bearing potential were not pregnant.

\subsection{End Points}

The primary measurements were the safety and efficacy in the reduction of the total number of lice observed on the subject's head and scalp after treatment assessed on Day 1, 8 and 15. An evaluation was performed at each subject's visit to determine whether their eyes were clear (value recorded 0) or red (value recorded 1). Additional assessments during the study for frequency of pruritus, erythema, presence of secondary infection, or excoriation were performed at each subject's visit using a four point scale detailed above [16-18].

\subsection{Statistical Analysis and Expression of the Results}

The sample size of 20 subjects per treatment group was a feasible sample size to provide descriptive statistics for the efficacy and safety parameters as no formal calculation of power or sample size was performed in this study. Fisher test and t-test were used when applicable to compare the efficacy of $1 \%$ Sodium Chloride spray product in eliminating head lice to that of Permethrin.

The following parameters were calculated: The proportion of subjects that were $100 \%$ free of live lice in each treatment group immediately post-dosing, and at the Day 8 and Day 15 visits and the proportion of subjects that required retreatment to be $100 \%$ free of lice at day 15 visit was recorded. For comparison, Fisher test and two sided 95\% confidence interval were performed on the difference in proportion between treatment groups. The numbers of live lice per head before the treatment and after each visit were also counted for the comparison between treatments. A p-value $<0.05$ was considered statistically significant. 


\section{Results}

\subsection{Demographic Characteristics of the Population}

Forty-two subjects participated in the study were assigned equally into each treatment group (Sodium Chloride spray or Permethrin). All females of child-bearing potential had negative pregnancy tests on Day 1 prior to their participation in this study. One subject in each treatment group completed the first treatment and first assessments but dropped out of the study prior to Day 8, and twenty subjects in each treatment group completed the study.

As shown in Table 1, there were no differences in age, race, and gender in the two treatment groups. Nearly half of all subjects had average texture to the hair $(47.6 \%$ in both treatment groups) but the curliness and length varied. The number of individuals with long and extra-long hair was higher in Sodium Chloride spray arm while curly hair occurred more in Permethrin arm.

Table 1. Each treatment group's subject demographic characteristics.

\begin{tabular}{|c|c|c|c|c|}
\hline & $\begin{array}{l}\text { Sodium } \\
\text { chloride }\end{array}$ & $\begin{array}{l}\text { Sodium } \\
\text { chloride }\end{array}$ & Permethrin & Permethrin \\
\hline & $\mathrm{n}=21$ & $\%$ & $\mathrm{n}=21$ & $\%$ \\
\hline \multicolumn{5}{|l|}{ Gender } \\
\hline Female & 18 & 85.71 & 20 & 95.24 \\
\hline Male & 3 & 14.29 & 1 & 4.76 \\
\hline \multicolumn{5}{|l|}{ Age } \\
\hline Mean & 15.57 & & 14.76 & \\
\hline Range & 6,43 & & 4,42 & \\
\hline \multicolumn{5}{|l|}{ Race } \\
\hline White & 18 & 85.71 & 19 & 90.48 \\
\hline American Indian & 2 & 9.52 & 0 & 0.00 \\
\hline Asian & 0 & 0.00 & 2 & 9.52 \\
\hline Black & 1 & 4.76 & 0 & 0.00 \\
\hline \multicolumn{5}{|l|}{ Ethnicity } \\
\hline Hispanic & 13 & 61.90 & 20 & 95.24 \\
\hline Non-Hispanic & 8 & 38.10 & 1 & 4.76 \\
\hline \multicolumn{5}{|l|}{ Hair length } \\
\hline Extra-long & 5 & 23.81 & 3 & 14.29 \\
\hline Long & 7 & 33.33 & 6 & 28.57 \\
\hline Medium & 7 & 33.33 & 11 & 52.38 \\
\hline Short & 2 & 9.52 & 1 & 4.76 \\
\hline \multicolumn{5}{|l|}{ Hair texture } \\
\hline Fine & 8 & 38.10 & 4 & 19.05 \\
\hline Average & 10 & 47.62 & 10 & 47.62 \\
\hline Coarse & 3 & 14.29 & 7 & 33.33 \\
\hline \multicolumn{5}{|l|}{ Hair curliness } \\
\hline Curly & 1 & 4.76 & 5 & 23.81 \\
\hline Wavy & 10 & 47.62 & 8 & 38.10 \\
\hline Straight & 10 & 47.62 & 8 & 38.10 \\
\hline
\end{tabular}

\subsection{Efficacy Assessment}

Immediately following treatment assessment (Figure 1) on Day 1, 79.2\% Sodium Chloride Spray subjects (16 of 21 ) and $66.7 \%$ Permethrin subjects (14 of 21) were lice free ( $\mathrm{p}>0.05$ ). At Day 8 visit, $25.0 \%$ subjects (5 out of 20) in Sodium Chloride Spray arm and $75.0 \%$ subjects (15 out of 20) in Permethrin arm required another course of treatment $(\mathrm{p}<0.05)$. Of those requiring retreatment at Day 8 visit, 3 of 5 Sodium Chloride spray subjects (60.0\%) were successfully treated as compared to 5 of 15 Permethrin subjects $(33.0 \%)$ ( $p>0.05$ ). Figure 2 presents the percentage of subjects free of lice at the end of the course of treatment (Day 15 visit), 85.0\% of the subjects in Sodium Chloride Spray arm and $45.0 \%$ of the subjects in Permethrin arm ( $\mathrm{p}<0.05)$.

The number of live lice presented prior to the initial treatment was comparable in both treatment groups (21.76 range 10 to 68 lice and 21.29 range 10 to $60, \mathrm{p}>0.05$, for Sodium Chloride Spray and Permethrin Crème Rinse, respectively). Immediately after treatment on Day 1, treatment failure subjects treated with Sodium Chloride Spray had $1.95 \pm 1.28$ lice as compared to $2.95 \pm 6.27$ in the Permethrin group ( $p>0.05$ ). At Day 8, the number of lice in subjects of the Sodium Chloride Spray treatment failure group was $1.40 \pm 2.19$ as compared to $2.95 \pm 2.98$ in the Permethrin group ( $\mathrm{p}>0.05)$.

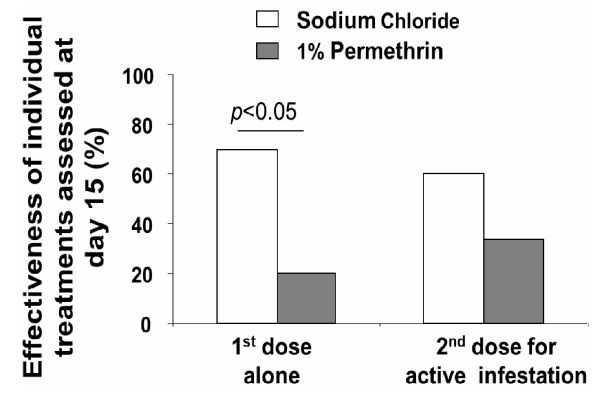

Figure 1. The percentage of subjects lice free after the 1st application and 2nd application for individuals needing a second treatment as assessed at Day 1 and 8 for Sodium Chloride Spray treatment arm of the study compared to the Permethrin treatment arm $(p<0.05)$.



Figure 2. The percentage of subjects lice free at Day 15 (completion of the study) for Sodium Chloride Spray treatment arm compared to Permethrin treatment arm $(p<$ 0.05). 
At Day 15, the number of lice observed in the treatment failure subjects of the Sodium Chloride Spray group was $0.55 \pm 1.5$ compared to $5.45 \pm 7.91$ in the Permethrin group $(\mathrm{p}<0.01)$. The summary of the efficacy results is presented in Table 2.

\subsection{Extent of Exposure}

Sixteen of the twenty-one subjects in the Sodium Chloride Spray arm received only the first application on day one (all were free of lice on day eight assessment) while another 6 subjects received a second application on day eight. One subject failed to return for the day eight assessment. This compares to six subjects receiving one application (subjects were free of lice on day eight assessment) and another 15 subjects receiving a second application of $1 \%$ Permethrin Crème Rinse on day eight. Again one subject failed to return for the day eight assessment.

\subsection{Adverse Events}

As presented in Table 3, the secondary characteristics such as pruritus, erythema, secondary infection, and excoriation of the lice infestation from Sodium Chloride Spray treated group were comparable to the control group. However, the improvement in pruritus in subjects treated with Sodium Chloride was consistently greater compared to Permethrin Crème Rinse (day one $1.14+1.1$ versus $0.71+0.49$; day $150.29+0.47$ versus $0.43+0.53$ for Sodium Chloride and Permethrin, respectively) however, these values were not statistically significantly different due to the number of subjects included in the study. One subject (white, Hispanic, 11 year old female with extra-long, fine, straight hair), with Sodium Chloride spray, reported a headache on Day 4 of the study, for which she self-administered one dose of ibuprofen $\left(\right.$ Motrin $\left.^{\circledR}\right)$. This adverse event was the only one reported in the study and she was a treatment failure.

\subsection{Safety Assessment}

No abnormal or significant differences in vital signs were measured for subjects in this study between the treatment groups. During the study, no serious adverse events were observed. Future studies are needed to fully assess adverse events as the number of subjects in the study was low.

\section{Discussion}

Traditional approaches to treat head lice infestations include using natural oils, nit combing and hair removal have resulted in low effectiveness or are undesirable [19]. Using OTC pediculicidal chemicals remains the most popular method for treating head lice infestations. Recent studies have shown a dramatic reduction in insecticidal activity because of increased resistance to the current insecticides [14,19]. With current pediculicides, it has been shown that resistance to popular "old" OTC products by head lice is genetically regulated $[12,20]$, suggesting that the use of these agents may become impractical, and more effective products should be considered to eliminate the troublesome parasites.

Table 2. Efficacy results observed for subjects in each treatment group at each treatment day.

\begin{tabular}{|c|c|c|}
\hline & Sodium chloride & Permethrin \\
\hline & $\mathrm{n}=21$ & $\mathrm{n}=21$ \\
\hline Pre-treatment Lice-mean & 21.76 & 21.29 \\
\hline Pre-treatment Lice—range & 10,68 & 10,60 \\
\hline Day 1 Post treatment Lice-mean & 1.95 & 2.95 \\
\hline Day 1 Post treatment Lice—-standard deviation & 1.28 & 6.27 \\
\hline Day 1 Post treatment Lice-95\% confidence interval & 0.55 & 2.68 \\
\hline Number subjects with NO Lice post-treatment & $16(76.2 \%)$ & $14(66.7 \%)$ \\
\hline Day 8 Pre Lice-mean & 1.30 & 4.25 \\
\hline Number subjects with NO Lice at Day 8 pre-treatment & $15(75 \%)$ & $5(25 \%)$ \\
\hline Number subjects no show at Day 8 & 1 & 1 \\
\hline Number subjects retreated & $5(25 \%)$ & $15(75 \%)$ \\
\hline Day 8 Post treatment Lice-mean & 1.40 & 2.95 \\
\hline Day 8 Post treatment Lice—-standard deviation & 2.19 & 2.98 \\
\hline Day 8 Post treatment Lice-95\% confidence interval & 1.92 & 1.51 \\
\hline Day 15 Lice-mean & 0.55 & 5.45 \\
\hline Day 15 Post treatment Lice-standard deviation & 1.50 & 7.91 \\
\hline Day 15 Post treatment Lice-95\% confidence interval & 0.66 & 3.47 \\
\hline
\end{tabular}


Table 3. Subject evaluation values for pruritus, erythema, secondary infection, excoriation, and eyes during each treatment period of the study.

\begin{tabular}{|c|c|c|c|c|c|c|c|c|c|c|}
\hline & \multicolumn{2}{|c|}{ Visit-1 Pre $^{a}$} & \multicolumn{2}{|c|}{ Visit-1 post treatment } & \multicolumn{2}{|c|}{ Visit-2 Pre $^{\mathrm{c}}$} & \multicolumn{2}{|c|}{ Visit-2 post treatment } & \multicolumn{2}{|c|}{ Visit-3 $^{e}$} \\
\hline & $\begin{array}{l}\text { Sodium } \\
\text { chloride } \\
(\mathrm{n}=21)\end{array}$ & $\begin{array}{l}\text { Permethrin } \\
\quad(\mathrm{n}=21)\end{array}$ & $\begin{array}{l}\text { Sodium } \\
\text { chloride } \\
(\mathrm{n}=21)\end{array}$ & $\begin{array}{l}\text { Permethrin } \\
\quad(\mathrm{n}=21)\end{array}$ & $\begin{array}{l}\text { Sodium } \\
\text { chloride } \\
(\mathrm{n}=20)\end{array}$ & $\begin{array}{l}\text { Permethrin } \\
(\mathrm{n}=20)\end{array}$ & $\begin{array}{c}\text { Sodium } \\
\text { chloride } \\
(\mathrm{n}=5)\end{array}$ & $\begin{array}{l}\text { Permethrin } \\
\quad(\mathrm{n}=15)\end{array}$ & $\begin{array}{l}\text { Sodium } \\
\text { chloride } \\
(\mathrm{n}=20)\end{array}$ & $\begin{array}{l}\text { Permethrin } \\
(\mathrm{n}=20)\end{array}$ \\
\hline \multicolumn{11}{|c|}{ Pruritus } \\
\hline Average & 1.14 & 0.71 & 0.36 & 0.29 & 0.43 & 0.57 & 0.11 & 0.17 & 0.29 & 0.43 \\
\hline std dev & 1.10 & 0.49 & 0.63 & 0.49 & 0.51 & 0.53 & 0.33 & 0.41 & 0.47 & 0.53 \\
\hline Range & 0.3 & 0.3 & 0.2 & 0.2 & 0.1 & 0.2 & 0.1 & 0.1 & 0.1 & 0.3 \\
\hline \multicolumn{11}{|c|}{ Erythema } \\
\hline Average & 0.14 & 0.00 & 0.10 & 0.00 & 0.05 & 0.00 & 0.00 & 0.00 & 0.00 & 0.00 \\
\hline std dev & 0.53 & & 0.45 & & 0.22 & & & & & \\
\hline Range & 0.1 & & 0.2 & & 0.1 & & & & & \\
\hline \multicolumn{11}{|c|}{ Infection } \\
\hline Average & 0.07 & 0.00 & 0.05 & 0.00 & 0.05 & 0.00 & 0.00 & 0.00 & 0.00 & 0.00 \\
\hline std dev & 0.27 & & 0.22 & & 0.22 & & & & & \\
\hline Range & 0.1 & & 0.1 & & 0.1 & & & & & \\
\hline \multicolumn{11}{|c|}{ Excoriation } \\
\hline Average & 0.14 & 0.00 & 0.10 & 0.00 & 0.05 & 0.00 & 0.00 & 0.00 & 0.00 & 0.00 \\
\hline std dev & 0.53 & & 0.45 & & 0.22 & & & & & \\
\hline Range & 0.2 & & 0.2 & & 0.1 & & & & & \\
\hline \multicolumn{11}{|c|}{ Eyes } \\
\hline Average & 0.00 & 0.00 & 0.00 & 0.00 & 0.00 & 0.00 & $\begin{array}{c}\text { Not } \\
\text { collected }\end{array}$ & 0.00 & 0.00 & 0.00 \\
\hline
\end{tabular}

a: Day 1 pretreatment assessment; ${ }^{\text {b}}$ : Day 1 post treatment; ${ }^{\text {c: }}$ Day 8 pretreatment; ${ }^{\text {d }}$ : Day 8 post treatment; ${ }^{\text {e }}$ Day 8 and 15 post treatment combined assessment for subjects completing study.

The effective treatment rate in this study of $1 \%$ Sodium Chloride Spray was superior to $1 \%$ Permethrin Crème Rinse immediately after the first application. This is a very important factor because parents, children, and infested individuals experience anxiety and stress upon observing live lice crawling on their scalp after treatment, which may lead to unexpected psychological trauma [1, 21]. The one hour treatment time of Sodium Chloride spray is a feasible length of time for an absence-of-lice condition to occur due to the highly effective results observed after one application. The study personnel commented regularly on the ease of application and use of the Sodium Chloride spray product over the 1\% Permethrin Crème rise product though this was not evaluated during the study. The second application of Sodium Chloride spray a week later also provides an identical response leading to superior results in comparison to the reference product. The lower rate of retreatment in the Sodium Chloride Spray group may also be an indication that may be the result of the ovicidal capacity of the product clinically. The in vivo ovicidal efficacy of Sodium Chloride Spray has yet to be determined, however, in vitro data have shown that ovicidal activity of gelled $10 \%$ Sodium Chloride formulation is greater than that of Permethrin and other tested chemicals [22]. The superior efficacy of Sodium Chloride Spray to 1\% Permethrin
Crème Rinse is important because Permethrin Crème Rinse is to be applied to towel dried hair requiring additional effort and time on the part of the users, whereas $1 \%$ Sodium Chloride spray can be directly applied to hair without any preparation providing added convenience and less effort in treatment. One can question the possible interference of moisture on subjects' hair in this study that may cause the reduction of Permethrin activity but in another Permethrin study [23], the product was applied to damp hair with $97 \%$ effectiveness, assessed two weeks post treatment, suggesting that the current poor activity of permethrin is most likely because of another reason $[12,15,24,25]$.

One of the suggested alternatives for the treatment of therapy-resistant head lice is the use of prescription products [19]. In addition to a greater level of toxicity, the economic burden of prescription products, the waiting time and cost for physician visits add to the inconvenience of this treatment option making a simple and inexpensive OTC product a more feasible treatment option. Another alternative OTC product on the market recently evaluated for efficacy has its active ingredient dimethicone [26-29]. The reported mechanism of action of dimethicone on lice [27] is consistent with observations reported by Fahmy et al. [22] in in-vitro studies performed here. The mechanism of action of Sodium Chlo- 
ride on lice is not fully known but similar rupture of the gastrointestinal tract in lice is observed with its application. Desiccation is suggested as the mode of action of Sodium Chloride which explains the one hour exposure to lice for its effects to occur. The ingredients in Sodium Chloride spray include Water, $1 \%$ Sodium Chloride and Poloxamer 188 (a surface active wetting agent to provide more efficient water and salt contact with the louse) as principle components.

The concentration of Sodium Chloride must be hypertonic to be effective against lice. Therefore, dilution of the active ingredient to hypotonic concentrations nullifies Sodium Chloride's activity against lice. In addition, Sodium Chloride must remain in contact with the louse in liquid form for an extensive period of time to be effective. The Poloxamer 188 is included in the product to facilitate Sodium Chlorides extended residence on the louse.

The present study demonstrates that $1 \%$ Sodium Chloride Spray is a simple application, and supplies an excellent medical alternative to the current recommended treatment for head lice to $1 \%$ Permethrin Crème Rinse. With the increase in the resistance of head lice to Permethrin developing across the country, $1 \%$ Sodium Chloride Spray is a great option for the treatment of head lice. The application of either Sodium Chloride Spray or Permethrin provides no safety concerns in the population treated. The lack of using a metal nit comb during the study was designed to provide a direct assessment of the two products. The study also provides an indirect assessment of ovicidal activity due to the day eight and fifteen posttreatment head lice evaluations which showed minimal reinfestation that would occur if nits survived the initial treatment. Use of a metal nit comb in therapy is likely to provide greater efficacy during therapy, but the extent of the improvement will need to be evaluated in the future.

\section{Acknowledgements}

Tec Laboratories Inc. provided funding for the study. The authors are deeply indebted to Tec Labs Inc. for their generous help in supplying their lice treatment product for the study. Other authors report no conflict of interest.

\section{REFERENCES}

[1] M. E. Falagas, D. K. Matthaiou, P. I. Rafailidis, G. Panos and G. Pappas, "Worldwide Prevalence of Head Lice," Emerging Infectious Diseases, Vol. 14, No. 9, 2008, pp. 1493-1494. doi:10.3201/eid1409.080368

[2] P. E. Fournier, J. B. Ndihokubwayo, J. Guidran, P. J. Kelly and D. Raoult, "Human Pathogens in Body and Head Lice,” Emerging Infectious Diseases, Vol. 8, No. 12, 2002, pp. 1515-1518. doi:10.3201/eid0812.020111

[3] K. Y. Mumcuoglu, S. Klaus, D. Kafka, M. Teiler and J.
Miller, "Clinical Observations Related to Head Lice Infestation," Journal of the American Academy of Dermatology, Vol. 25, No. 2, 1991, pp. 248-251. doi:10.1016/0190-9622(91)70190-D

[4] D. Taplin and T. L. Meinking, "Scabies, Lice, and Fungal Infections,” Primary Care, Vol. 16, No. 3, 1989, pp. 551576.

[5] M. Tebruegge, A. Pantazidou and N. Curtis, "What's Bugging You? An Update on the Treatment of Head Lice Infestation," Archives of Disease in Childhood-Education and Practice, Vol. 96, No. 1, 2010, pp. 2-8. doi:10.1136/adc.2009.178038

[6] K. Y. Mumcuoglu, T. A. Meinking, C. N. Burkhart and C. G. Burkhart, "Head Louse Infestations: The "No Nit" Policy and Its Consequences," International Journal of Dermatolog, Vol. 45, No. 8, 2006, pp. 891-896. doi:10.1111/j.1365-4632.2006.02827.x

[7] CDC, “Parasites-Lice-Head Lice Diagnosis,” 2011. http://www.cdc.gov/parasites/lice/head/diagnosis.html

[8] C. N. Burkhart and C. G. Burkhart, "Fomite Transmission in Head Lice," Journal of the American Academy of Dermatology, Vol. 56, No. 6, 2007, pp. 1044-1047. doi:10.1016/j.jaad.2006.10.979

[9] M. Takano-Lee, J. D. Edman, B. A. Mullens and J. M. Clark, "Transmission Potential of the Human Head Louse, Pediculus capitis (Anoplura: Pediculidae),” International Journal of Dermatolog, Vol. 44, No. 10, 2005, pp. 811816. doi:10.1111/j.1365-4632.2005.02418.x

[10] A. M. Downs, K. A. Stafford, L. P. Hunt, J. C. Ravenscroft and G. C. Coles, "Widespread Insecticide Resistance in Head Lice to the Over-the-Counter Pediculocides in England, and the Emergence of Carbaryl Resistance," British Journal of Dermatology, Vol. 146, No. 1, 2002, pp. 88-93. doi:10.1046/j.1365-2133.2002.04473.x

[11] T. L. Meinking, M. Vicaria, D. H. Eyerdam, M. E. Villar, S. Reyna and G. Suarez, "Efficacy of a Reduced Application Time of Ovide Lotion (0.5\% Malathion) Compared to Nix Creme Rinse (1\% Permethrin) for the Treatment of Head Lice,” Pediatric Dermatology, Vol. 21, No. 6, 2004, pp. 670-674. doi:10.1111/j.0736-8046.2004.21613.x

[12] K. S. Yoon, J. R. Gao, S. H. Lee, J. M. Clark, L. Brown and D. Taplin, "Permethrin-Resistant Human Head Lice, Pediculus capitis, and Their Treatment," Archives of Dermatology, Vol. 139, No. 8, 2003, pp. 994-1000. doi:10.1001/archderm.139.8.994

[13] CDC, "Malathion Frequently Asked Questions,” 2011. http://www.cdc.gov/parasites/lice/head/gen_info/faqs_ma lathion.html

[14] C. G. Burkhart and C. N. Burkhart, "Safety and Efficacy of Pediculicides for Head Lice,” Expert Opinion on Drug Safety, Vol. 5, No. 1, 2006, pp. 169-179. doi:10.1517/14740338.5.1.169

[15] AHRQ, "Guidelines for the Diagnosis and Treatment of Pediculosis capitis (Head Lice) in Children and Adults," 2011.

http://www.guideline.gov/content.aspx?id=12784\&search =head+lice+treatment 
[16] H. I. Katz, E. Gross, M. Buxman, S. E. Prawer, E. H. Schwartzel and J. R. Gibson, "A Double-Blind, Vehicle-Controlled Paired Comparison of Halobetasol Propionate Cream on Patients with Plaque Psoriasis,” Journal of the American Academy of Dermatology, Vol. 25, No. 6, 1991, pp. 1175-1178. doi:10.1016/0190-9622(91)70321-R

[17] K. Vie, A. Pons-Guiraud, P. Dupuy and H. Maibach, "Tolerance Profile of a Sterile Moisturizer and Moisturizing Cleanser in Irritated and Sensitive Skin,” Journal of the American Academy of Dermatology, Vol. 11, No. 3, 2000, pp. 161-164. doi:10.1053/ajcd.2000.7183

[18] D. Wilson and D. Nix, "Evaluation of a Once-Daily Moisturizer Used to Treat Xerosis in Long-Term Care Patients," Ostomy Wound Management, Vol. 51, No. 11, 2005, pp. 52-60.

[19] M. Lebwohl, L. Clark and J. Levitt, “Therapy for Head Lice Based on Life Cycle, Resistance, and Safety Considerations,” Pediatrics, Vol. 119, No. 5, 2007, pp. 965974. doi:10.1542/peds.2006-3087

[20] S. H. Lee, K.-S. Yoon, M. S. Williamson, S. J. Goodson, M. Takano-Lee, J. D. Edman, et al., "Molecular Analysis of KDR-Like Resistance in Permethrin-Resistant Strains of Head Lice, Pediculus capitis," Pesticide Biochemistry and Physiology, Vol. 66, No. 2, 2000, pp. 130-143. doi:10.1006/pest.1999.2460

[21] T. L. Meinking, L. Serrano, B. Hard, P. Entzel, G. Lemard, E. Rivera, et al., "Comparative in Vitro Pediculicidal Efficacy of Treatments in a Resistant Head Lice Population in the United States," Archives of Dermatology, Vol. 138, No. 2, 2002, pp. 220-224. doi:10.1001/archderm.138.2.220

[22] S. Fahmy, J. M. Christensen and L. Ma, "In Vitro and In Vivo Evaluation of Pesticidal and Ovicidal Activities of Chemical Pesticides of OTC Products, Home Remedies, Essential Oils and Other factors Associated with Efficacy of Treatment of Head Lice Infestations," Journal of Pharmaceutical Research and Clinical Practice, Vol. 2,
No. 1, 2012, pp. 1-13.

[23] D. Taplin, T. L. Meinking, P. M. Castillero and R. Sanchez, "Permethrin 1\% Creme Rinse for the Treatment of Pediculus humanus var Capitis Infestation," Pediatric Dermatology, Vol. 3, No. 4, 1986, pp. 344-348. doi:10.1111/j.1525-1470.1986.tb00538.x

[24] M. Kristensen, "Identification of Sodium Channel Mutations in Human Head Louse (Anoplura: Pediculidae) from Denmark," Journal of Medical Entomology, Vol. 42, No. 5, 2005, pp. 826-829. doi:10.1603/0022-2585(2005)042[0826:IOSCMI]2.0.CO; 2

[25] M. Kristensen, M. Knorr, A. M. Rasmussen and J. B. Jespersen, "Survey of Permethrin and Malathion Resistance in Human Head Lice Populations from Denmark," Journal of Medical Entomology, Vol. 43, No. 3, 2006, pp. 533-538. doi:10.1603/0022-2585(2006)43[533:SOPAMR]2.0.CO;2

[26] J. Huekelbach, D. Pilger, F. A. Oliveria, A. Khakban, L. Ariza and H. Feldmeier, "A Highly Efficacious Pediculicide Based Dimeticone: Randomized Observer Blinded Comparative Trial,” BMC Infectious Diseases, Vol. 8, 2008, pp. 115-124. doi:10.1186/1471-2334-8-115

[27] I. F. Burgess, “The Mode of Action of Dimeticone 4\% Lotion against Head Lice, Pediculus capitus," BMC Pharmacology, Vol. 9, 2009, pp. 3-11. doi:10.1186/1471-2210-9-3

[28] J. Huekelbach, A. Asenov, O. Liesenfeld, A. Mirmohammadsadegh and F. A. Oliveria, "A New Two Phase Dimeticone Pediculicide Shows High Efficacy in Comparative Bioassay,” BMC Dermatology, Vol. 9, 2009, pp. 12-18. doi:10.1186/1471-5945-9-12

[29] I. F. Burgess, P. N. Lee and G. Matlock, "Randomised, Controlled, Assessor Blind Trial Comparing 4\% Dimeticone Lotion with 0.5\% Malathion Liquid for Head Louse Infestation,” Plos One, Vol. 2, No. 11, 2007, pp. e1127e1134. doi:10.1371/journal.pone.0001127 